\title{
Marsdenia tenacissima extract suppresses tumor growth and angiogenesis in A20 mouse lymphoma
}

\author{
XINGBIN DAI $^{1^{*}}$, YANHUA JI ${ }^{2 *}$, PENGJUN JIANG $^{1}$ and XUEMEI SUN ${ }^{1}$ \\ ${ }^{1}$ Department of Hematology, Affiliated Hospital of Nanjing University of Traditional Chinese Medicine, Nanjing, \\ Jiangsu 210029; ${ }^{2}$ School of Life Science and Technology, China Pharmaceutical \\ University, Nanjing, Jiangsu 210009, P.R. China
}

Received May 14, 2015; Accepted September 27, 2016

DOI: $10.3892 / \mathrm{ol} .2017 .5831$

\begin{abstract}
Marsdenia tenacissima (MT), a traditional Chinese medicine, has been utilized in the treatment of a variety of malignant conditions for decades, but the underlying mechanism remains unclear. Angiogenesis, new blood vessel formation by nearby endothelial cells (ECs) from pre-existing vessels, plays a key role in cancer growth. In the present study, the effects of MT extract (MTE) on EC proliferation and apoptosis in vitro, and on A20 mouse lymphoma growth and angiogenesis in vivo were investigated. MTE exhibited an anti-proliferative effect on the ECs, with a half maximal inhibitory concentration of $11.91 \pm 0.24 \mu \mathrm{l} / \mathrm{ml}$. Acridine orange/propidium iodide staining indicated that cell apoptosis increased with MTE concentration. Flow cytometry revealed that the EC apoptosis rates induced by 0, 6.25, 12.5 and $25 \mu \mathrm{l} / \mathrm{ml}$ MTE were 4.8, 23.3, 49.8 and 92.3\%, respectively. In vivo, the volume and weight of the A20 solid tumors were significantly inhibited following administration of $300 \mu \mathrm{l}$ MTE per day for 14 days $(\mathrm{P}<0.05)$. MTE showed extended survivability and a satisfactory security. Subsequent to treatment with MTE, peritumorous angiogenesis was significantly reduced, with lower microvessel density $(\mathrm{P}<0.05)$ was quantified by hemotoxylin and eosin staining. Moreover, serum vascular endothelial growth factor, matrix metalloproteinase (MMP)-2 and MMP-9 expression at the protein level in the MTE-treated group, quantified using an ELISA, was significantly lower than that of the control $(\mathrm{P}<0.05)$. In a chick chorioallantoic membrane assay, 12.5 and $25 \mu \mathrm{l} / \mathrm{ml}$ MTE distinctly decreased the level of angiogenesis $(\mathrm{P}<0.05)$. In conclusion, MTE exhibited potent anti-lymphoma efficacy in vitro and this may be associated with its effects against tumor angiogenesis.
\end{abstract}

Correspondence to: Professor Xuemei Sun, Department of Hematology, Affiliated Hospital of Nanjing University of Traditional Chinese Medicine, 155 Hanzhong Road, Nanjing, Jiangsu 210029, P.R. China

E-mail: xuemeisun2014@yeah.net

${ }^{*}$ Contributed equally

Key words: Marsdenia tenacissima, antitumor, angiogenesis, herbal medicine

\section{Introduction}

Angiogenesis, the process of the formation of new blood vessels from pre-existing ones, plays a key role in the progression of solid tumors and hematological malignancies, such as lymphoma (1). Anti-angiogenesis can inhibit tumor growth and metastasis, and has become an appealing strategy for tumor therapy.

Traditional Chinese medicine (TCM) has been used for several thousand years, and has been suggested to aid the prevention and treatment of diseases such as cancer. Over the past 40 years, a number of TCM herbs have become a focus of interest globally; anticancer components have been isolated from medicinal herbs, which have been demonstrated to serve as modifiers of biological responses and as inhibitors of angiogenesis (2).

The stem of Marsdenia tenacissima (MT), also known as Marsdeniae tenacissimae (Roxb) Wight et Arn (family Asclepiadaceae), is well known as 'Tong-guan-teng' in TCM. Over the past 30 years, the antitumor effects of MT have aroused attention and passion from researchers. Previous studies have indicated that MT could inhibit proliferation and induce apoptosis in various cancer cell lines, such as HepG2/Dox, SGC 7901, Lewis lung carcinoma, U937, HL60, Jurkat, Raji and RPMI8226 (3-7). Studies in vitro revealed that MT exerts antitumor activity through different mechanisms, such as direct inhibition, inducing apoptosis and differentiation, and immunity adjustments (8). However, to date, few studies have focused on its antitumor effect in vivo, particularly in lymphoma cells. It is also unclear whether MT acts through other specific target(s), such as anti-angiogenesis.

The present study reports the growth inhibitory effects of MT extract (MTE) against murine B-cell lymphoma in vivo. Furthermore, the feasibility of MTE for treating lymphoma via anti-angiogenesis is also investigated.

\section{Materials and methods}

MTE (Xiaoiping injection; code, 200709121) was supplied by Nanjing Sanhome Pharmaceutical Co., Ltd., (Nanjing, Jiangsu, China). The preparation of plant extracts was performed according to the Drug Standards of the Ministry of Public Health of the People's Republic of China (9) and according to previously reported literature (10). MT bark and kibe powder 
(20 g with $40 \mathrm{ml} \mathrm{75 \%} \mathrm{ethanol)} \mathrm{were} \mathrm{refluxed} \mathrm{twice} \mathrm{for} 1.5 \mathrm{~h}$ in a hot water bath. Following vacuum concentration, the filtrate was degreased with $40 \mathrm{ml}$ petroleum ether $\left(60-90^{\circ} \mathrm{C}\right)$. The final extracts were partitioned with n-butanol twice to give n-butanol extract.

Cell lines and culture. Endothelial cells (ECs) were obtained from 10 newborn female C57BL/6 mice (1 week old; $1.5 \mathrm{~g}$ ) and were prepared as previously described (11). A20 cells, the mouse B-cell lymphoma cell line, were obtained from the Molecular Biology Laboratory of Jiangsu Province Hospital of TCM (Nanjing, Jiangsu, China). The two cell types were maintained in RPMI 1640 medium (Gibco; Thermo Fisher Scientific, Inc., Waltham, MA, USA) containing 10\% fetal bovine serum (FBS; Hangzhou Sijiqing Biological Engineering Materials Co., Ltd., Hangzhou, China) and $1 \%$ penicillin/streptomycin at $37^{\circ} \mathrm{C}$ in a humidified air atmosphere with $5 \% \mathrm{CO}_{2}$.

Animal models and ethics. A total of $20 \mathrm{female} \mathrm{BALB/c}$ mice (weighing 18-22 g; 4-6 weeks old), purchased from the College of Veterinary Medicine of Yangzhou University (Yangzhou, Jiangsu, China), were maintained in a pathogen-free environment in the Center of Laboratory Animals, Jiangsu Province Hospital of TCM and were provided with free access to food and water. All animal experiments were approved by the Animal Ethics Committee of the Chinese Medicine Hospital of Jiangsu province, and all mice were handled strictly in accordance with the National Institutes of Health Guidelines for the Care and Use of Laboratory Animals.

\section{3-(4,5-dimethylthiazol-2-yl)-2,5-diphenyl-2H-tetrazolium} bromide (MTT) assay. The cytotoxicity of MTE against ECs was determined by MTT assay. Briefly, the cells were seeded in 96-well plates, with $1 \times 10^{4}$ cells/well in $200 \mu \mathrm{l}$ of RPMI 1640 medium supplemented with $10 \% \mathrm{FBS}$ at $37^{\circ} \mathrm{C}$ in a humidified atmosphere with $5 \% \mathrm{CO}_{2}$, and then were either left untreated or were treated with MTE $(0,6.25,12.5,25$ and $50 \mu \mathrm{l} / \mathrm{ml})$. Following co-incubation for $48 \mathrm{~h}, 20 \mu \mathrm{l}$ of MTT solution [10 $\mathrm{mg} / \mathrm{ml}$ in phosphate-buffered saline (PBS)] was added to each well for an additional 4-h incubation. Subsequently, the medium was discarded and the formed dark-blue formazan crystals in intact cells were dissolved in $200 \mu$ l dimethyl sulfoxide. Absorbance was measured at $490 \mathrm{~nm}$ with a microplate reader (Spectra Max 340PC; Bio-Rad Laboratories, Hercules, CA, USA).

Acridine orangelpropidium iodide (AO/PI) staining. ECs $\left(5 \times 10^{4}\right)$ treated with indicated concentrations $(0,6.25,12.5$ and $25 \mu \mathrm{l} / \mathrm{ml}$ ) of MTE were incubated at $37^{\circ} \mathrm{C}$ with $5 \% \mathrm{CO}_{2}$ for $48 \mathrm{~h}$. At the end time point, each cell culture was centrifuged at $800 \times g$ and $4^{\circ} \mathrm{C}$ and rinsed twice with normal saline (NS), and then resuspended in NS and stained with $\mathrm{AO} / \mathrm{PI}$ solution $(100 \mu \mathrm{g} / \mathrm{ml} \mathrm{AO}$ and $100 \mu \mathrm{g} / \mathrm{ml} \mathrm{PI})$. Images were acquired using a confocal laser scanning microscopy (LSM510; Zeiss GmbH, Jena, Germany).

Quantification of apoptosis by flow cytometry. The percentage of apoptotic cells was examined by double staining with Annexin-V-fluorescein isothiocyanate (FITC) and PI. Briefly, $\sim 1 \times 10^{5}$ cells/well, untreated or treated with MTE $(0,6.25,12.5$ and $25 \mu \mathrm{l} / \mathrm{ml}$ ), were seeded into 6-well plates and incubated at $37^{\circ} \mathrm{C}$ for $48 \mathrm{~h}$. Subsequently, whole cells were harvested and treated with $5 \mu \mathrm{l}$ Annexin V-FITC for $30 \mathrm{~min}$. Prior to being analyzed by fluorescence-activated cell sorting (Beckman Coulter EPICS Altra; Beckman Coulter, Inc., Brea, USA), $5 \mu 1$ of PI was added to each tube.

Animal tumor models and treatment. In total, $6.0 \times 10^{5}$ viable A20 cells in $100 \mu 1$ PBS were used to establish solid tumor models after subcutaneous injection into the right forelimbs of the BALB/c mice. At $24 \mathrm{~h}$ post-injection, 20 mice were randomly divided into two groups (10 mice per group), namely the control and MTE groups. Following the onset of a visible solid tumor, the mice in the MTE group were administered daily intraperitoneal injections of $300 \mu \mathrm{l}$ MTE for 14 consecutive days, and the mice in the control group were administered $300 \mu \mathrm{l}$ NS in the same manner. During administration, subcutaneous injection tumors were measured with Vernier callipers every 3 days. The tumor volume (V) was calculated according to the following formula: $\mathrm{V}=0.52 \mathrm{x}$ length $\mathrm{x}$ width $^{2}$. A total of 5 mice from each group were sacrificed by cervical dislocation $24 \mathrm{~h}$ after the last administration. The eyeball was removed and 0.8-1.0 ml blood was collected and then centrifuged at $800 \mathrm{x} g$ for $15 \mathrm{~min}$ at $4^{\circ} \mathrm{C}$. The supernatant was collected and stored at $-80^{\circ} \mathrm{C}$ in a refrigerator for the enzyme-linked immunosorbent assay (ELISA) of vascular endothelial growth factor, matrix metalloproteinase (MMP)-2 and MMP-9 suing commercial kits (cat. nos. EK0955 and EK1302; Elabscience Biotechnology Co., Ltd., Wuhan, China). The tumor tissues were dissected and weighed, then collected for further analysis.

In order to further investigate the anti-lymphoma activity of MTE in the murine model, the survival times of another 10 mice were recorded (5 mice per group). Side effects during the experiment, including anepithymia, ruffling of fur, altered mental state, behavioral changes, reaction to stimulation and weight loss, were also observed.

For the tumor peritumorous angiogenesis experiments, the animal tumor model was developed as aforementioned. The experimental animals were grouped separately and administered with $300 \mu \mathrm{l}$ MTE or vehicle for 7 days. When a tumor mass was detectable visually, the mice were sacrificed by cervical dislocation. Tumor tissues were excised and images of peritumorous blood vessels were captured.

Microvessel density (MVD) analysis. Tumor tissues fixed with $10 \%$ neutral-buffered formalin were processed and subjected to histopathological analysis with hematoxylin and eosin (HE) staining for the measurement of MVD using a Lawrence and Mayo Lynx Reg microscope, as previously described (12).

ELISA. The level of serum VEGF was determined by the ELISA, using specific commercial kits (cat. no. EK0327; Elabscience Biotechnology Co., Ltd.) according to the manufacturer's protocols. Briefly, the plasma samples obtained by centrifugation from the control or MTE-treated mice were pipetted into ELISA plates coated with polyclonal antibody specific for mouse VEGF (cat. no. 500-P131; 1:250; PeproTech, Inc., Rocky Hill, NJ, USA). The captured antibody was then detected by a biotinylated monoclonal antibody against 
A

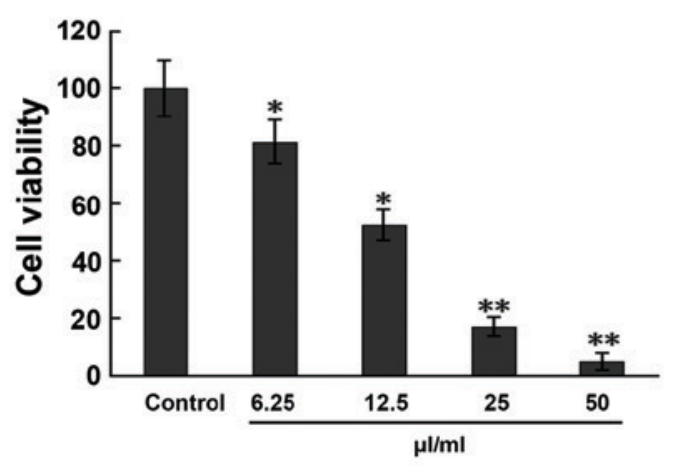

B

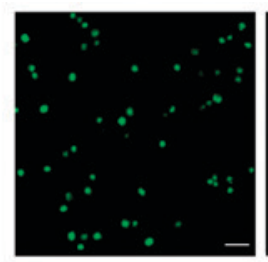

C

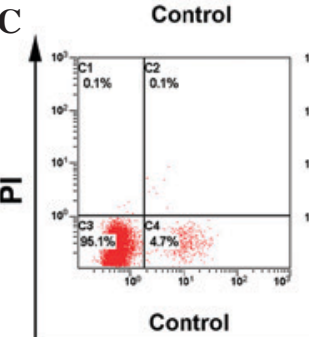

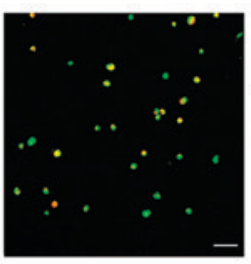

$6.25 \mu \mathrm{l} / \mathrm{ml}$

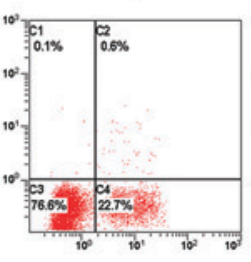

$6.25 \mu \mathrm{l} / \mathrm{ml}$

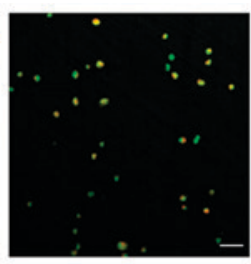

$12.5 \mu \mathrm{l} / \mathrm{ml}$

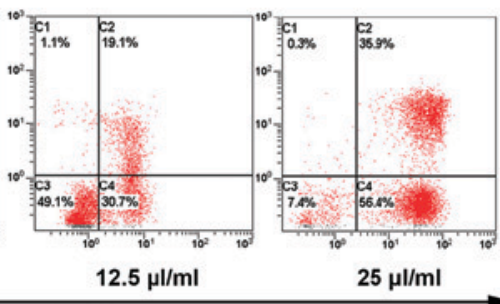

Annexin-V

Figure 1. Apoptosis and anti-proliferative effects induced by MTE on ECs. (A) ECs were treated with MTE $(0,6.25,12.5,25$ and $50 \mu 1 / \mathrm{ml})$ for $48 \mathrm{~h}$. The cell viability was analyzed by the 3-(4,5-dimethylthiazol-2-yl)-2,5-diphenyl-2H-tetrazolium bromide assay. (B) Following incubation with different concentrations of MTE $(0,6.25,12.5,25 \mu \mathrm{l} / \mathrm{ml})$ for $48 \mathrm{~h}$, the cells were stained with acridine orange/PI solutions and observed under a confocal laser scanning microscope. Green, jacinth and red spots represent living, apoptotic and necrotic cells, respectively (x200 magnification). Bar, $10 \mu \mathrm{m}$. (C) ECs were incubated with the indicated doses of MTE for $48 \mathrm{~h}$. After being stained with Annexin V-fluorescein isothiocyanate and PI, apoptotic cell death (\%) was determined with flow cytometry. Lower left, Annexin $\mathrm{V}^{-} / \mathrm{PI}^{-}$cells (normal); lower right, Annexin $\mathrm{V}^{+} / \mathrm{PI}^{-}$cells (early apoptosis); upper right, Annexin $\mathrm{V}^{+} / \mathrm{PI}^{+}$cells (late apoptosis); and upper left, Annexin $\mathrm{V}^{-} / \mathrm{PI}^{+}$cells (necrosis). Results are represented as the mean \pm standard deviation from three separate experiments. ${ }^{*} \mathrm{P}<0.05$; ${ }^{* *} \mathrm{P}<0.001$ vs. control. MTE. Marsdenia tenacissima extract; EC, endothelial cell; PI, propidium iodide.

mouse VEGF (cat. no. PL03F1637M; PL Laboratories Inc., Vancouver, BC, Canada). Bound anti-VEGF was quantified at $450 \mathrm{~nm}$ after addition of streptavidin peroxidase and substrate. The expression of MMP-2 and MMP-9 was detected using the ELISA kit (Wuhan Boster Biological Technology, Ltd., Wuhan, China) following the manufacturer's protocols.

Chick chorioallantoic membrane (CAM) assay. The anti-angiogenesis activity of MTE was further verified by the CAM assay, which is another reliable model for angiogenesis studies (13). Embryos (27 days old) were randomized into two groups: The control and MTE groups (10 embryos per group). In brief, a pore ( $\sim \mathrm{mm}$ in diameter) was punctured in the shell concealing the air sac and $100 \mu \mathrm{l}$ MTE or NS was carefully infused into the CAM. Next, the eggs were resealed with transparent film and incubated for a further 2 days at $37^{\circ} \mathrm{C}$ in $60 \%$ humidity. Subsequent to incubation and treatment with MTE, images of the blood vessels on the surface of the CAM were captured using a canon digital camera (Canon, Inc., Tokyo, Japan) in the treated and untreated eggs.

Statistical analysis. All statistical analyses were performed using the SPSS 17.0 software package (SPSS, Inc., Chicago, IL, USA). All data, with the exception of overall survival experiments are expressed as the mean \pm standard deviation. Survival was estimated using the Kaplan-Meier product limit method. Comparison between groups was assessed using a one-way analysis of variance followed by Tukey's multiple comparison test. $\mathrm{P}<0.05$ was considered to indicate a statistically significant difference.

\section{Results}

Anti-proliferative and apoptosis-inducing effect of MTE on ECs. The effect of MTE on EC viability was assessed using the
MTT assay. As shown in Fig. 1A, the inhibitory effect of MTE on the ECs occurred in a concentration-dependent manner. Compared with the control, cell viability decreased 18.6, 47.5, 85.9 and $97.9 \%$, respectively, in the $6.25,12.5,25$ and $50 \mu \mathrm{l} / \mathrm{ml}$ treatment groups. Calculated by the Bliss method, the half maximal inhibitory concentration $\left(\mathrm{IC}_{50}\right)$ value of MTE was $11.91 \pm 0.24 \mu \mathrm{l} / \mathrm{ml}$ for the ECs.

Based on the results of the cytotoxicity of MTE, further studies were performed. The morphological examination of ECs by the AO/PI staining method showed that the apoptosis rate markedly increased with increasing MTE concentration, with statistical significance among the indicated concentrations (Fig. 1B). Similar results were obtained from the Annexin-V/PI staining assay. In Fig. 1C, compared with the control, the percentages of ECs undergoing early apoptosis $\left(\right.$ Annexin- $\left.\mathrm{V}^{+}, \mathrm{PI}\right)$ and late apoptosis $\left(\right.$ Annexin- $\left.\mathrm{V}^{+}, \mathrm{PI}^{+}\right)$in the MTE-treated group increased in a concentration-dependent manner to $4.8,23.3,49.8$ and $92.3 \%$, respectively.

MTE inhibits murine A20 lymphoma growth in vivo without any side effects. The results of a scatter graph of solid tumor weight are presented in Fig. 2A. The tumor weights of the control and MTE groups were $3.06 \pm 0.78$ and $0.77 \pm 0.25 \mathrm{~g}$, respectively, and a significant difference $(\mathrm{P}<0.01)$ was shown between the two groups (Fig. 2B). Fig. $2 \mathrm{C}$ shows that the tumor volume of the MTE group was smaller than that of the control group $(\mathrm{P}<0.05)$. Furthermore, the survival time in the MTE group was markedly prolonged compared with that in the control group $(\mathrm{P}<0.05)$ (Fig. 2D). During administration, no side effects, such as bad appetite, reduced activity, stunted response, colorless fur or weight loss, were observed in the MTE group.

MTE inhibits angiogenesis. Angiogenesis is required for growth, as well as for the expansion of solid tumors, 
A

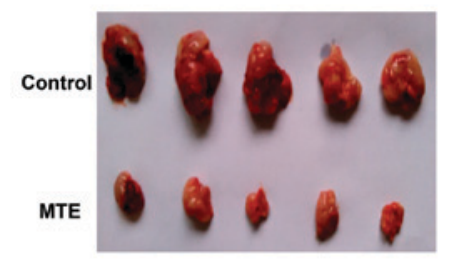

C

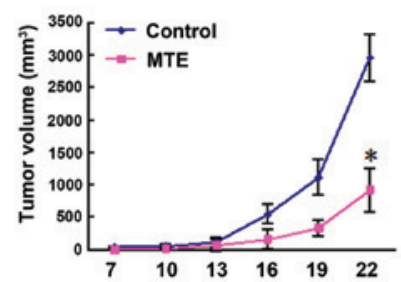

B

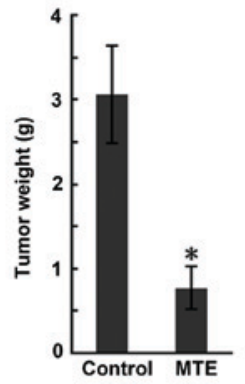

D

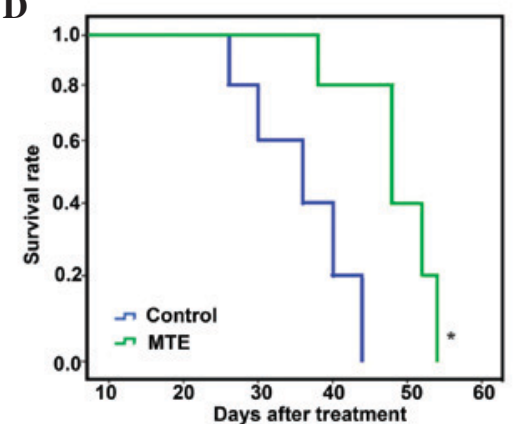

Figure 2. Effect of MTE on progression of A20 lymphoma in mice after 14 days of treatment. Solid tumors were induced in female BALB/c mice by injecting A20 cells subcutaneously into the right forelimb region. A daily intraperitoneal injection of $300 \mu 1$ MTE or normal saline for 14 consecutive days was administered to tumor-bearing mice. (A) Representative tumor images after treatment. (B) The average tumor weight of the tumor-bearing mice. (C) Inhibition of tumor growth by MTE, as measured by tumor volume. (D) Kaplan-Meier survival curve. Results are expressed as the mean \pm standard deviation from two-independent experiments. ${ }^{*} \mathrm{P}<0.05$ and ${ }^{* *} \mathrm{P}<0.001$ vs. control. MTE. Marsdenia tenacissima extract.

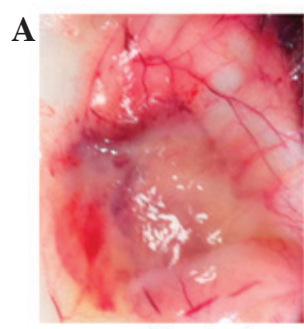

Control

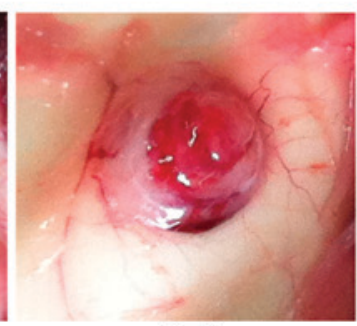

MTE

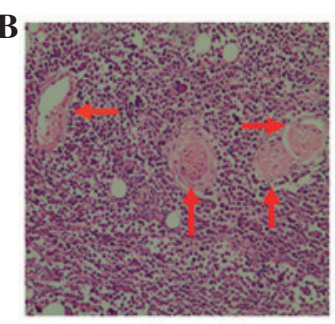

Control

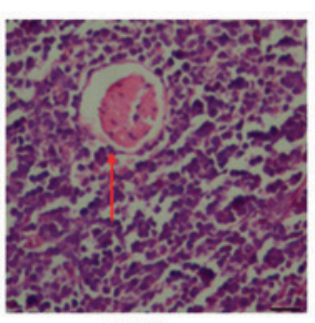

MTE

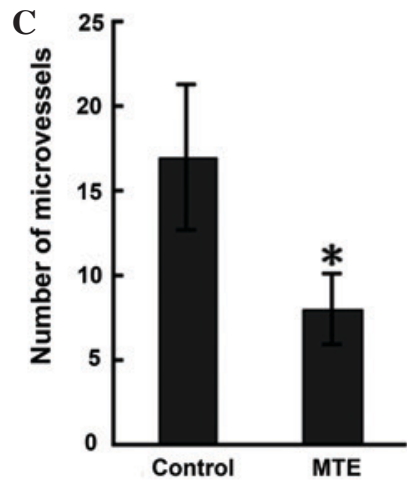

Figure 3. MTE reduces neovascularization. Solid tumors were induced in female BALB/c mice by injecting A20 cells subcutaneously into the right forelimb region. (A) A daily intraperitoneal injection of $300 \mu \mathrm{l}$ MTE for 7 consecutive days was administered to the tumor-bearing mice. When a tumor mass was detectable visually, tumor tissues were excised and images of the peritumorous blood vessel were captured. (B) Representative photomicrographs showing the hematoxylin and eosin staining of A20 tumor tissue sections in control and MTE-treated mice (x200 magnification). Bar, $10 \mu \mathrm{m}$. (C) The microvessel density (x200 magnification). Results are expressed as the mean \pm standard deviation from two independent experiments. ${ }^{*} \mathrm{P}<0.05$ vs. control. MTE. Marsdenia tenacissima extract.

particularly those at $1-2 \mathrm{~mm}$ in diameter (14). In the present study, the angiopreventative effect of MTE on the surface of mouse subcutaneous tumors was verified. Unexpectedly, MTE significantly reduced peritumorous angiogenesis compared with no treatment in the tumor-bearing mice (Fig. 3A). Similar results were obtained from HE staining (Fig. 3B). The MTE-treated mice exhibited a significantly lower MVD than the control mice (Fig. 3C).

MTE reduces the production of VEGF, MMP-2 and MMP-9. VEGF is one of the most potent and specific promoters of angiogenesis, and it acts by stimulating ECs to form new vessels (15). ELISA was performed to evaluate the production of VEGF in the tumor-bearing mice, and the results are presented in Fig. 4A. MTE reduced the VEGF level compared with the control.

MMPs, a family of at least 24 zinc-dependent endopeptidases that degrade the basement membrane and all protein components of the extra-cellular matrix, are hypothesized to play an essential role in angiogenesis (16). In particular, MMP-2 and MMP-9 play vital roles in cancer cell invasion and metastasis, as the two MMPs are abundantly expressed in various malignant cancer cells (17). In the present study, ELISA data in serum showed MMP-2 and MMP-9 expression in the MTE group (42.01 \pm 4.12 and $13.87 \pm 1.43 \mathrm{ng} / \mathrm{ml}$, respectively), but higher expression in the control group $(80.56 \pm 8.64$ and $25.73 \pm 2.31 \mathrm{ng} / \mathrm{ml}$, respectively). MTE significantly decreased the levels compared with the control (Fig. 4B and C). 
A

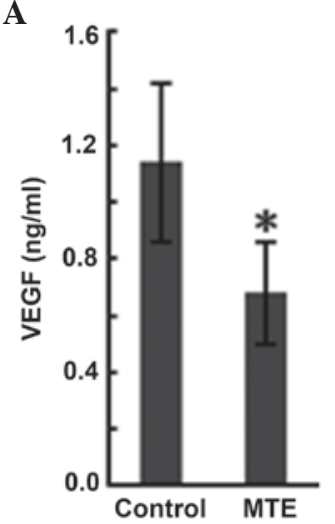

B

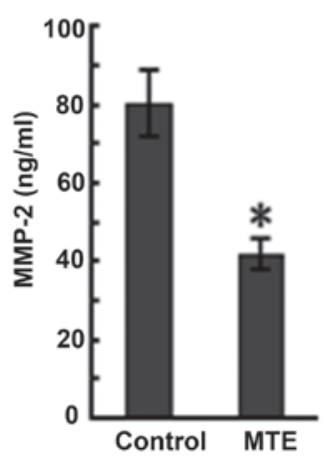

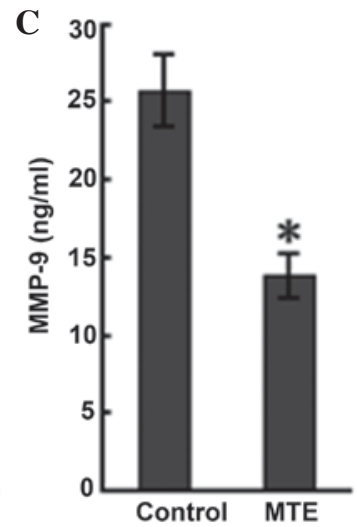

Figure 4. MTE reduces the production of VEGF and MMPs in tumor-bearing mice. (A) MTE inhibited the expression of VEGF in the serum of A20 inoculated mice. (B) MTE inhibited the expression of MMP-2 in the serum of A20 inoculated mice. (C) MTE inhibited the expression of MMP-9 in the serum of A20 inoculated mice. Results are expressed as the mean \pm standard deviation from two-independent experiments ( $\mathrm{n}=5$; " $\mathrm{P}<0.05$ vs. control). MTE. Marsdenia tenacissima extract.

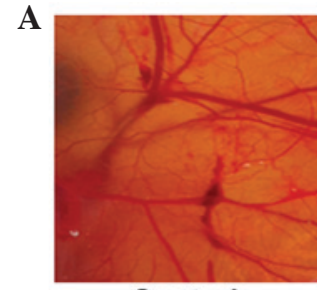

Control

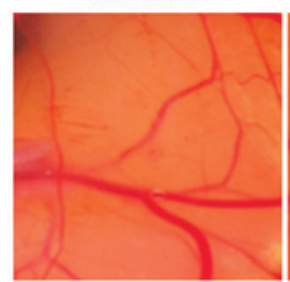

$12.5 \mu \mathrm{l} / \mathrm{ml}$

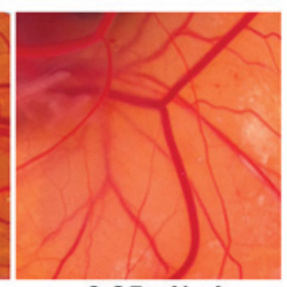

$6.25 \mu \mathrm{l} / \mathrm{ml}$

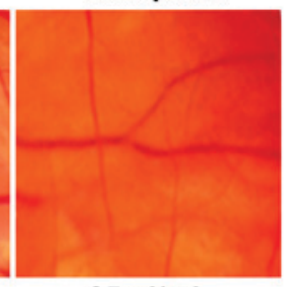

$25 \mu \mathrm{l} / \mathrm{ml}$

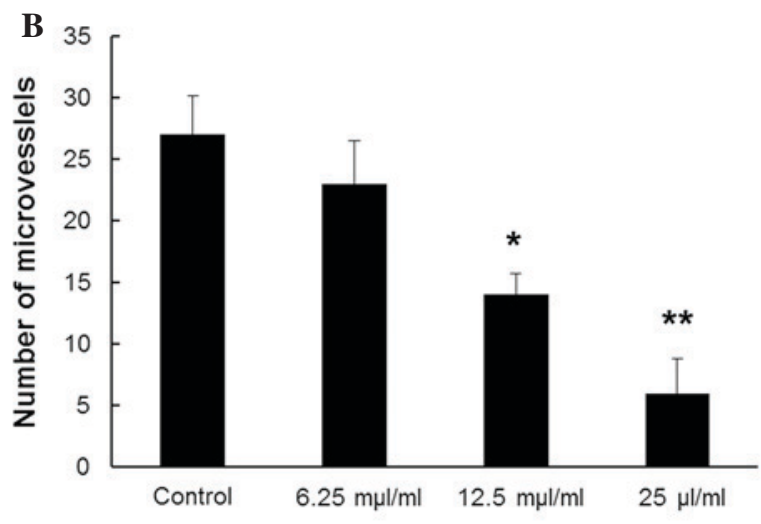

Figure 5. Angiopreventive effect of MTE in vivo by CAM assay. (A) $100 \mu \mathrm{l} \mathrm{MTE}$ or normal saline was infused into the CAM and incubated at $37^{\circ} \mathrm{C}$ in $60 \%$ humidity for 2 days. Images of the blood vessels on the surface of the CAM were captured. Representative CAM photos treated with various concentrations $(0,6.25,12.5,25 \mu \mathrm{l} / \mathrm{ml})$ of MTE. (B) The number of blood vessels was quantified manually in a circular perimeter $0.25 \mathrm{~cm}$ from the center. The experiments were performed twice. Data are expressed as the mean \pm standard deviation (error bars) $\left(\mathrm{n}=10 ;{ }^{*} \mathrm{P}<0.05 ;{ }^{* *} \mathrm{P}<0.01\right.$ vs. control). MTE. Marsdenia tenacissima extract; CAM, chick chorioallantoic membrane.

Inhibitory effects of MTE on angiogenesis in an in vivo CAM assay. A CAM assay was performed to directly examine the effect of MTE on angiogenesis and vascular development in vivo. As indicated in Fig. 5A, compared with the untreated vehicle control, CAM treated with MTE showed markedly fewer newly formed blood vessels. For quantitative analysis, new blood vessel branches in a circular area $(5 \mathrm{~mm}$ in diameter) surrounding the filter paper disc were counted. Fig. 5B shows that the MVD was dose-dependently reduced with MTE concentration $(\mathrm{P}<0.05)$, whereas a non-significance difference was observed between the control and $6.25 \mu \mathrm{l} / \mathrm{ml}$ MTE $(\mathrm{P}>0.05)$.

\section{Discussion}

The present study showed that MTE suppressed the growth and induced the apoptosis of ECs in vitro. The in vivo antitumor effect of MTE against A20 murine B-cell lymphoma model system was evident by the extended survival times.
In addition, MTE resulted in decreased angiogenesis when compared with the angiogenesis found on the surface of untreated tumor-bearing mice. The MVD in the tumor sections of the treated animals was decreased significantly compared with that of the control group. Furthermore, a reduction in VEGF, MMP-2 and MMP-9 expression in the serum was also observed in the treated group. The anti-angiogenesis activity of MTE was further assessed by the CAM assay.

The solid tumor model system is the most reliable and representative of major histological types of cancer, thereby providing the rapid action of drug delivery (18). MVD measurements are widely used as a surrogate marker in pathological specimens and tumor models in order to determine the disease prognosis $(10,19)$. VEGF regulates vascular formation through the initiation of vessel growth, the incorporation of hematopoietic and endothelial progenitor cells into the developing vasculature, and the inhibition of EC apoptosis (20). VEGF is the well-studied initial factor of angiogenesis $(21,22)$. Increased MMP expression renders tumor cells capable of 
digesting essential tissue barriers, particularly basement membranes that line blood vessels, thereby promoting cell motility (23). MMP-2 and MMP-9 are also able to regulate angiogenesis directly $(24,25)$. In the present study, MTE reduced the density of microvessels in the implanted A20 tumors and inhibited VEGF expression in the tumor-bearing mice. The results also indicated that MMP-2 and MMP-9 were important factors involved in the inhibition of angiogenesis by MTE. This indicates that MTE has potent anti-lymphoma activity and that the efficacy of anti-angiogenesis may be one of the possible underlying mechanisms of tumor inhibition. To the best of our knowledge, this is the first study in which the antitumor activity and the anti-angiogenesis potency of MTE were evaluated in a mouse tumor model system.

EC proliferation is the key step for the onset and progression of angiogenesis (26), and the induction of EC death could be a strategy for anti-angiogenic therapies (27). In the present study, MTE showed promising anti-proliferative potency against ECs with an $\mathrm{IC}_{50}$ of $11.91 \pm 0.24 \mu \mathrm{l} / \mathrm{ml}$ in the MTT assay. A similar result was found in a recent study, which demonstrated that MTE was highly effective at inhibiting cell proliferation in human umbilical vein ECs (HUVECs) by blocking the cell cycle progression from G1 to S phase (28). In addition, MTE has been shown to inhibit HUVEC migration and prevent vascular formation through VEGF suppression in vitro (22). Notably, the inhibition of EC proliferation in vitro and the suppression of angiogenesis in vivo appeared to be associated to the induction of EC apoptosis.

The process of angiogenesis is regulated by a series of positive and negative factors. Further study is required to investigate which step of VEGF signal transduction pathways in A20 cells can be taken as the acting target of the antagonists for MTE. Additional animal experiments and further clinical trials are also required to understand whether MTE inhibits angiogenesis through other signaling pathways.

In conclusion, the present study showed that MTE suppressed tumor growth and reduced angiogenesis in A20 mouse lymphoma. MTE may represent a natural anti-angiogenic target for lymphoma therapy.

\section{Acknowledgements}

The authors would like to thank Ms. Shuangshuang Wang, Jiangsu Province Hospital of TCM, for assisting with the histopathological experiments. In the process of a previous study on the synergic effect and mechanism on Burrkitt lymphoma induced by a combination of Xiaoaiping injection and chemotherapeutics based on Aurora kinase A (batch no, 81603456) supported by the National Natural Science Foundation, it was observed that decreased angiogenesis around tumors was shown in MTE treated mice; therefore the present study was subsequently carried out.

\section{References}

1. Ribatti D, Nico B, Crivellato E, Roccaro AM and Vacca A: The history of the angiogenic switch concept. Leukemia 21: 44-52, 2007.

2. Sun Y: The role of Chinese medicine in clinical oncology. Chin J Integr Med 20: 3-10, 2014.
3. $\mathrm{Hu} Y J$, Shen XL, Lu HL, Zhang YH, Huang XA, Fu LC and Fong WF: Tenacigenin B derivatives reverse P-glycoprotein-mediated multidrug resistance in HepG2/Dox cells. J Nat Prod 71: 1049-1051, 2008.

4. Li MQ, Shen JH, Xu B, et al: The mechanism of laboratory research for xiaoaiping treating SGC-7901 gastric carcinoma cellular strains. J Interventional Radiology 10: 228-231, 2001. (In Chinese)

5. Liang XH, Gao GH, Zhou XL, et al: The anti-angiogenic effect of Xiaoaiping injection on Lewis lung carcinoma in C57 mice. Chin Clin Oncol 15: 689-692, 2010. (In Chinese)

6. Li D, Ou YJ, Li CP, et al: Marsdensia tenacissima induces apoptosis of human U937, HL60 leukemic cells. Chin J Biochem Pharm 29: 33-37, 2008. (In Chinese)

7. Chen B, Li CP, Chen JH, et al: Effect of extract from Marsdenia tenacissima on Jurkat, Raji and RPMI8226 cells in vitro. Chin J of Biochem Pharmc 30: 174-177, 2009. (In Chinese)

8. Fang YQ and Sun XM: Review on Marsdenia tenacissima's chemical components and their antitumor mechanism. Chin J of Biochem Pharmac 32: 165-167, 2011. (In Chinese)

9. Editorial Committee of the pharmacopoeia of People's Republic of China: The pharmacopoeia of People's Republic of China, Part 1. Chemical Industry Press, Beijing, China, pp442-443, 2005. (In Chinese)

10. Yao Q, Lu TL, Mao CQ, et al: Determination of total saponins and tenac issoside-B in bark and kibe of Marsdenia tenacissima. Anhui Med and Pharmac J 14: 36-38, 2010 (In Chinese).

11. Zhang M, Tang H, Guo Z, An H, Zhu X, Song W, Guo J, Huang $X$, Chen T, Wang J and Cao X: Splenic stroma drives mature dendritic cells to differentiate into regulatory dendritic cells. Nat Immunol 5: 1124-1133, 2004.

12. Prabhakar BT, Khanum SA, Shashikanth S and Salimath BP: Antiangiogenic effect of 2-benzoyl-phenoxy acetamide in EAT cell is mediated by HIF-lalpha and down regulation of VEGF of in-vivo. Invest New Drugs 24: 471-478, 2006.

13. Staton CA, Reed MW and Brown NJ: A critical analysis of current in vitro and in vivo angiogenesis assays. Int J Exp Pathol 90: 195-221, 2009.

14. Folkman J: Proceeding: Tumor angiogenesis factor. Cancer Res 34: 2109-2113, 1974.

15. Ferrara N, Gerber HP and LeCouter J: The biology of VEGF and its receptors. Nat Med 9: 669-676, 2003.

16. Jackson C: Matrix metalloproteinases and angiogenesis. Curr Opin Nephrol Hypertens 11: 295-299, 2002.

17. Kang MS, Oh JS, Kang IC, Hong SJ and Choi CH: Inhibitory effect of methyl gallate and gallic acid on oral bacteria. J Microbiol 46: 744-750, 2008.

18. Talmadge JE, Singh RK, Fidler IJ and Raz A: Murine models to evaluate novel and conventional therapeutic strategies for cancer. Am J Pathol 170: 793-804, 2007.

19. Prabhakar BT, Khanum SA, Jayashree K, Salimath BP and Shashikanth S: Anti-tumor and proapoptotic effect of novel synthetic benzophenone analogues in Ehrlich ascites tumor cells. Bioorg Med Chem 14: 435-446, 2006.

20. Ellis LM and Hicklin DJ: VEGF-targeted therapy: Mechanisms of anti-tumor activity. Nat Rev Cancer 8: 579-591, 2008.

21. Folkman $\mathrm{J}$ : What is the evidence that tumors are angiogenesis dependent? J Natl Cancer Inst 82: 4-6, 1990.

22. Hanada D and Folkman J: Patterns and emerging mechanisms of the angiogenic switch during tumorigenesis. Cell 86: 353-364, 1996.

23. Egeblad M and Werb Z: New functions for the matrix metalloproteinases in cancer progression. Nat Rev Cancer 2: 161-174, 2002.

24. Bergers G, Brekken R, McMahon G, Vu TH, Itoh T, Tamaki K, Tanzawa $\mathrm{K}$, Thorpe $\mathrm{P}$, Itohara $\mathrm{S}$, Werb Z and Hanahan D: Matrix metalloproteinase-9 triggers the angiogenic switch during carcinogenesis. Nat Cell Biol 2: 737-744, 2000.

25. Coussens LM, Fingleton B and Matrisian LM: Matrix metalloproteinase inhibitors and cancer: Trials and tribulations. Science 295: 2387-2392, 2002.

26. Risau W: Mechanisms of angiogenesis. Nature 386: 671-674, 1997.

27. Folkman J: Angiogenesis and apoptosis. Semin Cancer Biol 13: 159-167, 2003

28. Huang Z, Lin H, Wang Y, Cao Z, Lin W and Chen Q: Studies on the anti-angiogenic effect of Marsdenia tenacissima extract in vitro and in vivo. Oncol Lett 5: 917-922, 2013. 